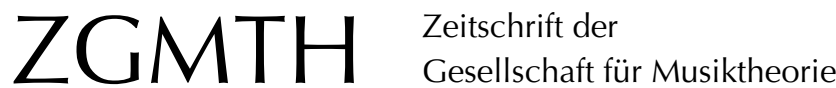

Petersen, Peter (2013): Absicht oder Zufall? Über Terzen- und andere Intervallketten in Brahms' 4. Sinfonie samt einer neuen Deutung des Zitats im Variationen-Finale. ZGMTH 10/1, 35-46. https://doi.org/10.31751/710

(C) 2013 Peter Petersen

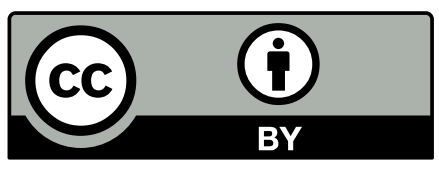

Dieser Text erscheint im Open Access und ist lizenziert unter einer Creative Commons Namensnennung 4.0 International Lizenz.

This is an open access article licensed under a Creative Commons Attribution 4.0 International License.

veröffentlicht / first published: 06/07/2014

zuletzt geändert / last updated: 19/02/2016 


\section{Absicht oder Zufall?

\author{
Über Terzen- und andere Intervallketten in Brahms' 4. Sinfonie \\ samt einer neuen Deutung des Zitats im Variationen-Finale
}

Peter Petersen

ABSTRACT: Dass das Hauptthema von Brahms' 4. Sinfonie aus Terzenketten besteht, ist schon bald nach der UA gewürdigt worden. Später wurde dann eine bestimmte Terzenkette im Finale als Anspielung auf den ersten Satz identifiziert. Unbemerkt blieb bisher, dass Brahms zugleich mit den Terzen auch eine Quartenkette hören lässt und somit die Interdependenz diatonischer Intervallzirkel - aus Sekunden, Terzen und Quarten - kompositorisch demonstriert. Darüber hinaus lässt sich analytisch nachweisen, dass die Anspielung im Finale nicht auf den Anfang der Sinfonie, sondern auf eine 20 Takte umfassende Sektion im Umkreis der Reprise des ersten Satzes bezogen ist.

Die Terzenketten, auf denen das Hauptthema im Kopfsatz von Brahms' 4. Sinfonie basiert ( $h$-g-e-c-a-fis-dis-h und e-g-h-d-f-a-c), und die Wiederaufnahme eben solcher Terzenketten im Finale derselben Sinfonie (e-c-a-fis-dis-h-g-e... in T. 233 und T. 241) gehören zu den heute allgemein anerkannten analytischen Befunden. Schon 1897 hatte Hugo Riemann ${ }^{1}$ das Thema des ersten Satzes auf das Modell der Terzenkette zurückgeführt und zudem auf die Nähe der Hauptthementerzen zu dem e-Moll-Arioso Behold and see aus dem 2. Teil von Händels Messiah hingewiesen (Anfang: [e-h]-e-c-a-fis-dis-h-g). 1908 machte Alfred Heuß² - bezugnehmend auf Riemann - auf eine weitere Terzenkette aufmerksam, die Brahms natürlich gekannt hat: Takt 78ff. im fis-Moll-Adagio aus Beethovens Hammerklavier-Sonate Op. 106 (ces-as-f-des-b-g-dis-h-gis-e-cis-ais-fis-dis-hisgis-e-cis-a-fis-dis-h-gis-eis-cis). 1912 ergänzte Max Kalbeck ${ }^{3}$ diese Angaben durch den Verweis auf Brahms' eigenes Op. 1, dessen Scherzo wie die 4. Sinfonie ebenfalls in eMoll steht und in dem einmal (bei T. 48) zehn Töne in eine Terzenkette eingebunden sind ( $h$-g-e-c-a-fis-dis-h-g-e). Arnold Schönberg ${ }^{4}$ war, soweit ich sehe, dann 1933 der erste, der den Zitatcharakter im Finale von Brahms' Vierter benannt hat, wo - wie erwähnt achttönige Terzenketten vorkommen.

1 Riemann 1897.

2 Heuß 1907-08.

3 Kalbeck 1904-1914, hier Bd. 3, 462f. Auf eine weitere Terzenstelle bei Brahms hat Bernard Jacobson aufmerksam gemacht: Im Finale des Klavierquartetts c-Moll op. 60 folgt ab Takt 110 eine Reihe fallender Terzen: $h$-gis-e-c-a-f-d-h-g-e-c-a-f-d-h (1977, 149).

4 Vgl. Finscher 1990, insb. 489. 
Mittlerweile sind weitere auffällige Terzenketten in Brahms' Vierter entdeckt worden. Sieht man von den Varianten des Hauptthemas im ersten Satz ab - die Terzengänge sind in ihrer Substanz unverändert (z. B. T. 19 und T. 273: aufgelöste Oktaven, Durchgänge, Wechselnoten; T. 157, T. 169 und T. 173: Vorhalte) -, so kommen Terzenketten in der Begleitung des zweiten Themas hinzu (T. 65: cis/ais-ais/fis-fis/d-d/h-h/g usw., analog auch T. 309) ${ }^{5}$, außerdem ein Terzenfall im Abgang des Fanfarenmotivs in der Durchführung (T. 190: $c$-as-f- $d$-h-g-es-c, analog auch T. 208 und T. 375). Während der zweite Satz keine Terzenketten aufweist, gibt es im dritten Satz zwei Stellen, die wohl als Anspielung an die Terzen des ersten Satzes gemeint sind (T. 27 und T. 305: h/d-d/f-f/a-a/c, T. 48 und analog T. 243: es-c-a-fis-d-h-g). Im vierten Satz ist eine zweimalige Terzenkette anzuführen, die - anders als die erwähnten Terzenfälle gegen Ende des Finales - die ursprüngliche Transpositionsstufe benutzt und die zudem wegen ihres Klangcharakters als deutliche Allusion auf den Sinfoniebeginn zu werten ist (T. 38 und T. 62: h-g-e-c-a-fis-dis).

Längst bekannt ist auch der Zusammenhang von Terzenfolge und Sekundfortschreitung, der die Interdependenz der Hauptthemen von Kopfsatz und Finale begründet. Dieser Zusammenhang ist weniger spektakulär als manche Interpreten es darstellen. ${ }^{6}$ Wie Christian Martin Schmidt bereits deutlich gemacht hat ${ }^{7}$, stellen Sekunden-, Terzen- und Quartengänge drei Modi dar, mit denen das diatonisch-heptatonische Tonsystem abgebildet werden kann. Dass in der Musiktheorie seit der Antike die Präsentation des Tonbestands als Skala bevorzugt wird, hängt mit der Musikpraxis (Stimmung der Instrumente) zusammen, wäre aber tonsystematisch nicht zwangsläufig geboten. Wird die Folge über den 7. Ton hinaus fortgesetzt, tritt das System in einen diatonischen Zirkel ein. Dessen Merkmal besteht (im Gegensatz zu chromatischen Zirkeln ${ }^{8}$ ) darin, dass stets verschiedene Intervallklassen beteiligt sind: große und kleine Sekunden, große und kleine Terzen, reine und übermäßige Quarten. Am Beispiel des Bestands A, H, C, D, E, F, G seien die drei Darstellungsarten des diatonisch-heptatonischen Tonvorrats veranschaulicht:

$\begin{array}{lllllllll}\text { Stufen: } & 1 & 2 & 3 & 4 & 5 & 6 & 7 & \text { (8) } \\ \text { Sekunden: } & \text { A } & \text { H } & \text { C } & \text { D } & \text { E } & \text { F } & \text { G } & \text { (A) } \\ \text { Terzen: } & \text { A } & \text { C } & \text { E } & \text { G } & \text { H } & \text { D } & \text { F } & \text { (A) } \\ \text { Quarten: } & \text { A } & \text { D } & \text { G } & \text { C } & \text { F } & \text { H } & \text { E } & \text { (A) }\end{array}$

Abb. 1: Darstellungsarten des diatonisch-heptatonischen Tonvorrats durch Sekund-, Terz- und Quartgang

5 Vetter, der alle Terzenstrukturen im 1. Satz präzise beschrieben hat, kann überdies plausibel machen, dass auch das ankündigende Fanfarenmotiv in Takt 53f. mit seiner Tonfolge ...cis-ais-fis-fis$d$... die begleitende Terzenkette von Takt 57ff. teilweise vorwegnimmt (1913-1914, 83).

6 Z. B. Kross 1997, vgl. besonders Bd. 2, $917 \mathrm{ff}$.

7 Schmidt 1971, $18 \mathrm{ff}$.

8 Im vierten Satz der 4. Sinfonie setzt Brahms einmal zu einem chromatischen Sekundenzirkel in Verbindung mit einem Quartenzirkel an, biegt den Vorgang aber dann so um, dass es am Ende bei einer erweiterten diatonischen Kadenz bleibt (Variation 11, T. 94-97, Diskant: e-dis-d-cis-c-h-ais-a-g-fis-e; Bass: $h$-e-a-d-g-c-fis-h-e). 
Bei Endlosfolgen lassen sich aus jeder der drei Darstellungsformen des Tonbestands die anderen beiden ableiten:

A HCDEFGAHCDEFGAHCDEFGA HCDEFGA... (Sekunden)

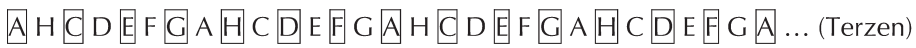

AHCDEFGAHCDEFGA HCDEFGACDEFGA... (Quarten)

A CEGHDFACEGHDFACEGHDFACE G H D F ... (Terzen)

ACEGHDFACEGHDFACEGHAFCEGHDA... (Sekunden)

ACEGHDFAEGHEFACEGHEACEGHDFA... (Quarten)

A D G CFHEA D G C F HE A D G C F HE A D G C F HE A ... (Quarten)

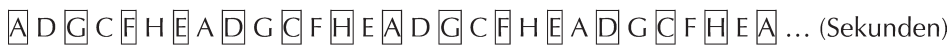

ADGCFHEA D C F H A G C F H A D C F H A ... (Terzen)

Abb. 2: Jeweilige Herleitung einer Darstellungsart aus den übrigen

Dass Brahms diese Zusammenhänge bewusst waren, kann jener Stelle im vierten Satz entnommen werden, bei der das Chaconne-Thema (e-fis-g-a-...) aus den Tönen einer Terzenkette herauszuhören ist $\left(e^{2}-c^{2}-a^{1}-f i s^{1}-d i s^{1}-h-g^{2}-e^{2}-c^{2}-a^{1}-f i s^{1}-d i s^{1}\right)$. Interessant ist nun, dass hier in Takt 233ff. gleichzeitig mit den Terzen auch Quartenketten erklingen, die den diatonischen Quartenzirkel in sieben Stufen darstellen. ${ }^{9}$ Der Tonbestand ist jetzt gemäß harmonisch e-Moll organisiert (E, Fis, G, A, H, C, Dis, E), woraus sich diese realen Tonhöhen einer Quartenfolge ergeben: $h^{1}-e^{2}-a^{2}-d i s^{3}-g^{2}-c^{3}-f i s^{3}$ (Beispiel 1).

Die tonsystematisch bedingte Verwandtschaft diatonischer Sekunden-, Terzen- und Quartenzirkel tritt auch im 1. Satz bei bestimmten Variantenbildungen des Hauptthemas zu Tage. Das aus fallenden, durch Legatobögen verbundenen Terzen zusammengesetzte Thema wird vielfältig variiert - u.a. auch durch eingefügte Vorhaltsnoten. Da Brahms das Hauptmotiv metrisch stets auftaktig setzt $(h \mid g)$, fungiert ein das Terzintervall ausfüllender Ton, sofern er auf schwerem Taktteil erklingt ( $h \mid \mathrm{a}-\mathrm{g}$ ), als frei eintretender Vorhalt (und nicht etwa als Durchgangsnote ${ }^{10}$ ). In Beispiel 2 habe ich die entsprechenden Motivvarianten außer in der originalen Notation auch in einer Umschrift eingetragen, die von der - zu Brahms' Zeit nicht mehr gebräuchlichen - Notationsform für harmonische Vorhalte (kleine Noten) Gebrauch macht. So werden die hinter den melodischen Sekunden bzw. Septimen liegenden Terzenketten wieder deutlich (T. 157): $d$-b-g-es-c-a-fis- $d$. Etwas später (T. 169), nachdem sich die Vorhaltsvarianten im Charakter verändert haben

9 Auf die Verbindung von Terzen- und Quartenketten in den Takten 233ff. und $241 \mathrm{ff}$. hat Knapp aufmerksam gemacht (1997, 188, Anm. 42).

10 So Mäckelmann etwas unpräzise in seiner ansonsten fundierten Analyse von Brahms' 4. Sinfonie (1991, 41). 


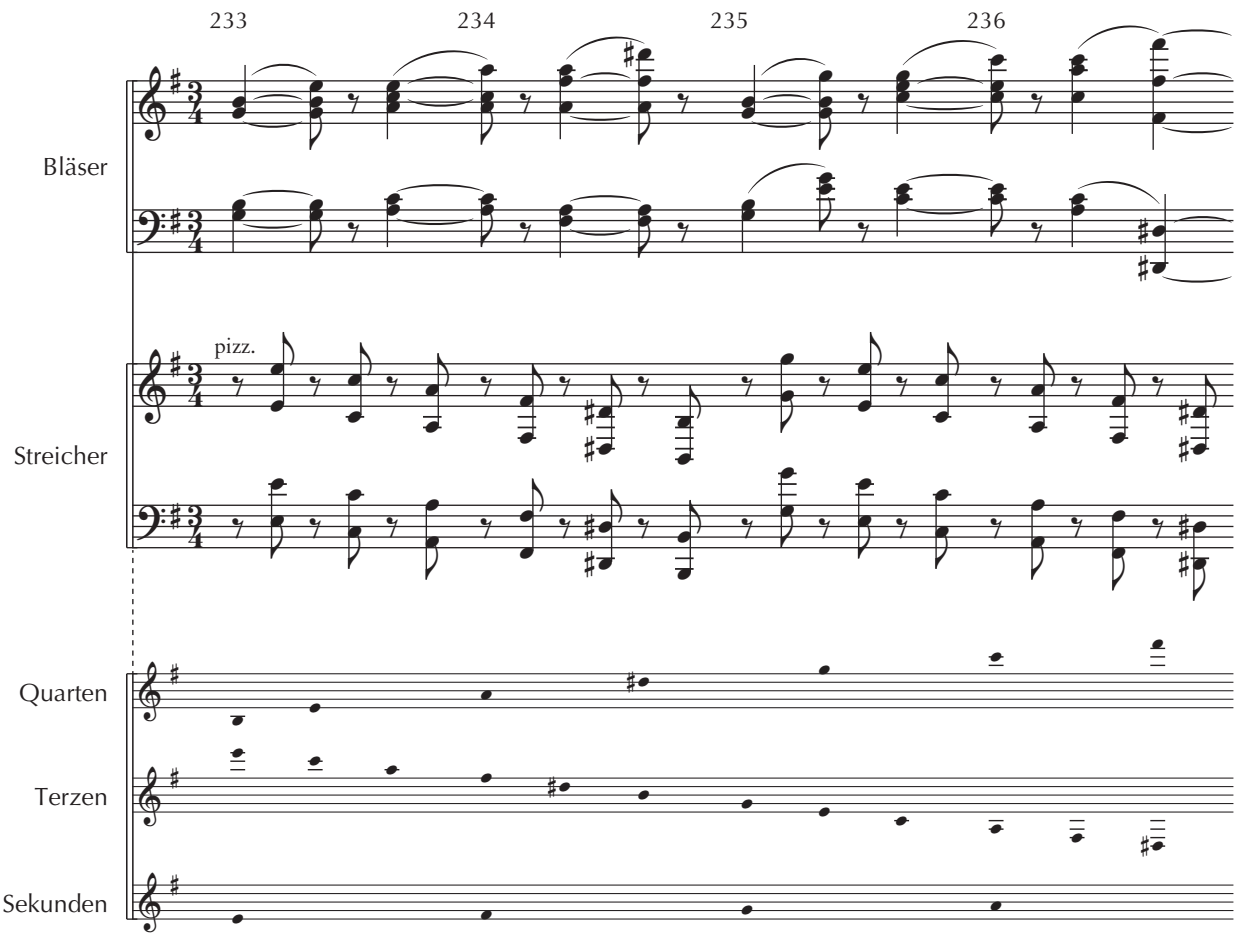

Beispiel 1: Johannes Brahms, 4. Sinfonie e-Moll op. 98, Finale, T. 233-236

(von legato zu akzentuiert, von Piano zu Forte) und zudem mit einer gegenläufigen Bassstimme angereichert worden sind, ergeben sich sogar drei diatonische Intervallzirkel: einer aus Terzen $(f$-des- $b$-ges-es-c-as- $f$-des), einer aus Sekunden ( $f$-es-des-c- $b$-asges-f-es-des-c usw.) und einer aus Quarten ( $b$-es-as-des). Dies gilt natürlich nur unter der Voraussetzung, dass in Tonhöhenklassen gedacht wird, von der konkreten Oktavlage der Töne also abgesehen wird. Brahms selbst demonstriert dies bereits beim Hauptthema, wo z. B. der vierte Terzton, der eigentlich ein zweigestrichenes c sein müsste, ins höhere Register $\left(c^{3}\right)$ springt (Beispiel 2).

Solche Oktavversetzungen könnten eventuell die Ursache dafür sein, dass manche Ton- und Motivbeziehungen in Brahms' Vierter nicht erkannt wurden. So blieb im Scherzo ein Vorauszitat des Finale-Themas lange Zeit unbemerkt und findet noch heute in aktuellen deutschen Werkeinführungen keine Erwähnung, weil es hinter Oktavversetzungen verborgen ist. Es handelt sich im Finale um den Tonleiteraufstieg bis zum fünften Ton des Chaconne-Themas, der in der Regel ja als übermäßige Quarte, in Takt 213 und dann vor allem in der Coda auch als verminderte Quinte erscheint: e-fis-g-a-ais bzw. efis-g-a-b. Alan Walker hat als erster darauf hingewiesen, dass es im 3. Satz eine Akkordfolge gibt, die im Kern einen Sekundgang $\left(a^{3}-h^{1}-c^{4}-d^{2}-e s^{4}\right)$ enthält, der im Finale auf die Coda-Fassung des Chaconne-Themas vorausdeutet (Beispiel 3). ${ }^{11}$ 


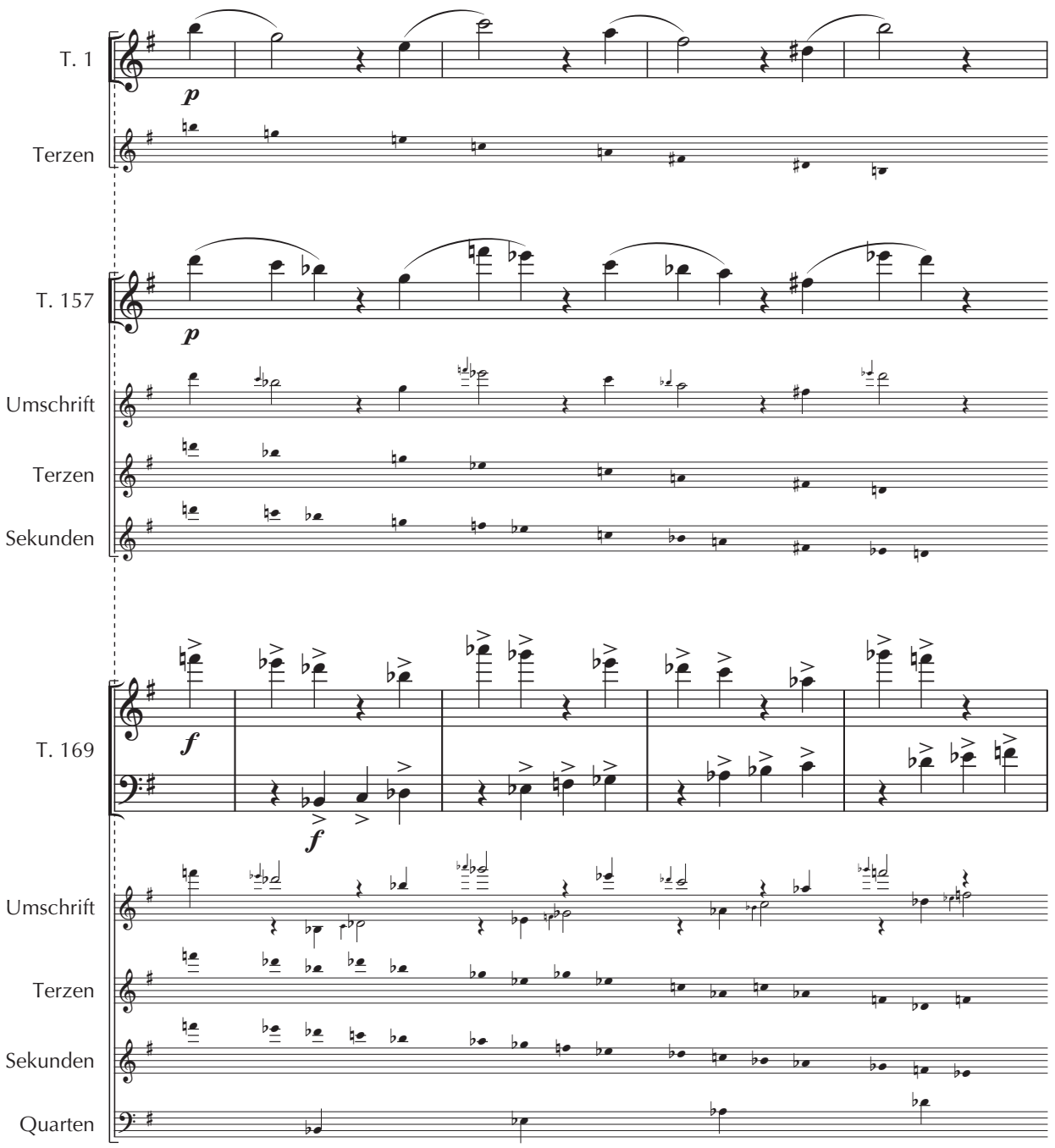

Beispiel 2: Johannes Brahms 4. Sinfonie e-Moll op. 98, 1. Satz, Hauptthema, Vorhaltsvarianten, T. 1-4, T. 157-160 und T. 169-172

11 Vgl. Walker 1962, 76:»[...] a pre-hearing of the passacaglia idea towards the end of the third movement." Das Notenbeispiel bei Walker zeigt dann allerdings das Thema in der Fassung von Takt 1 und nicht die Coda-Version. Auch Knapp geht auf dieses Vorauszitat im 3. Satz ein, allerdings ohne auf Walker zu verweisen $(1997,191)$. Horton $(1968,60 f$.) führt nur die erste Stelle mit dem f-a-c-disAkkord (3. Satz T. 93 ff.) an, nicht aber den Stufengang a- $h$-c- $d$-es gegen Ende des 3. Satzes (T. 317). 

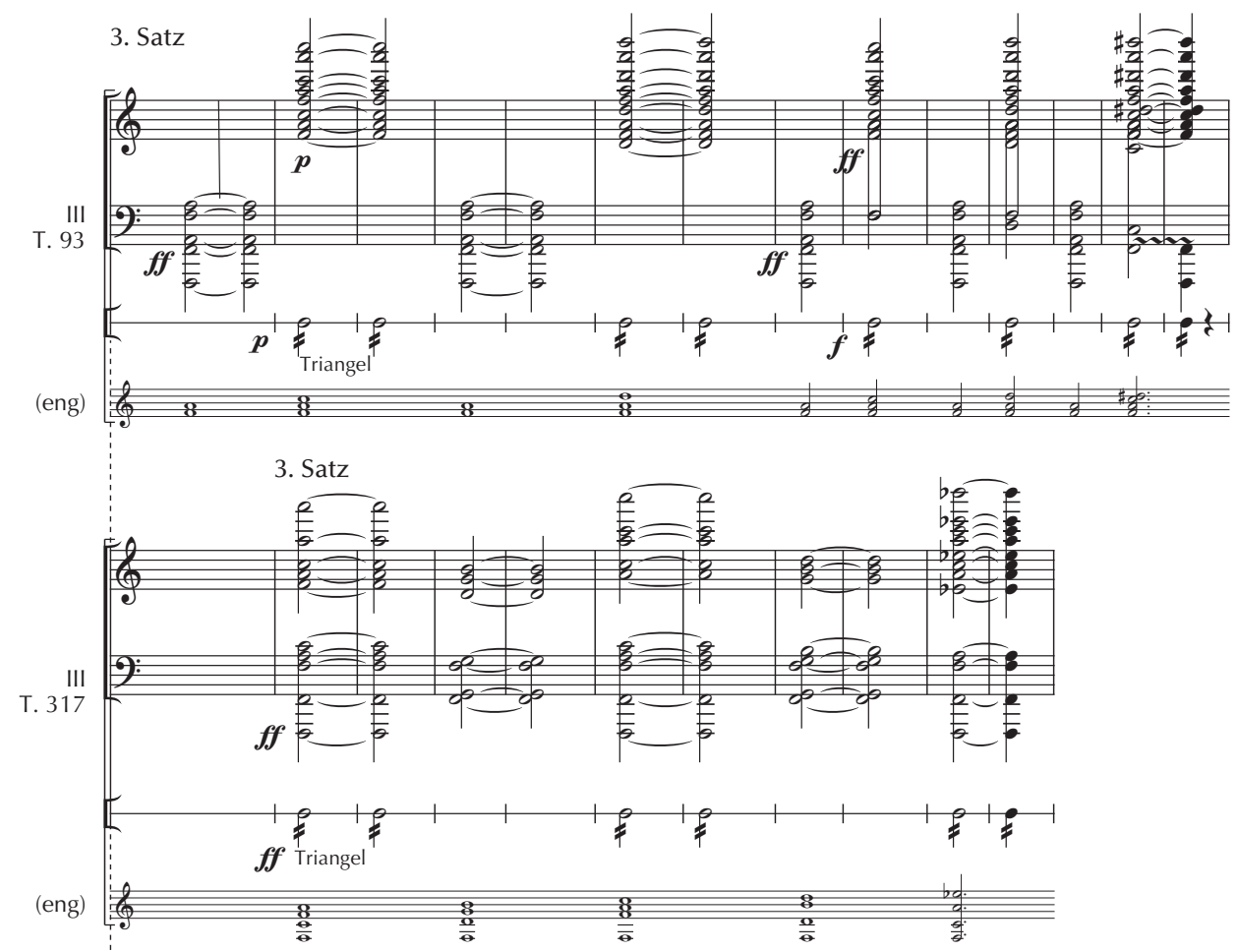

IV

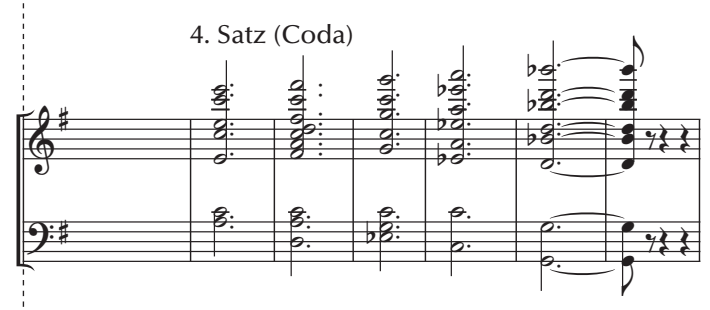

4. Satz (Coda)

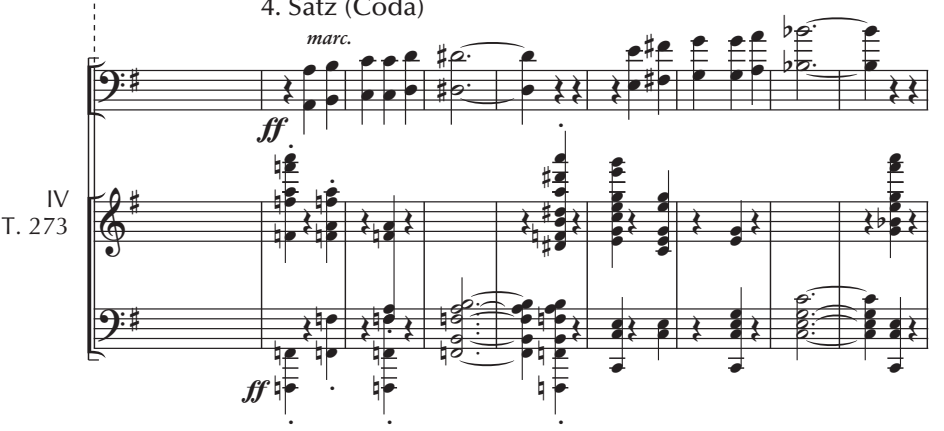

Beispiel 3: Johannes Brahms, 4. Sinfonie e-Moll op. 98, Ankündigung des Chaconne-Themas im 3. Satz, T. 93-107 und T. 317-326 
Ich habe in Beispiel 3 je zwei Stellen aus dem 3. und 4. Satz zusammengestellt, die wohl keinen Zweifel darüber zulassen, dass diese Anspielung vom Komponisten intendiert war. Brahms lässt dies erst beim zweiten Mal, also in der Reprise des Hauptteils des Scherzos, wirklich kenntlich werden (T. 317-326; vgl. das System seng`), während das erste Mal die Sekundenkette fehlt, wobei allerdings das Zielintervall, die übermäßige Quarte, doch schon vorkommt (T. 93-107; vgl. wieder das System sengı). In der Coda des 4. Satzes setzt das Thema ein, als handelte es sich um eine weitere Reprise. Allerdings bricht es mit dem fünften Ton ab, und zwar auf der verminderten Quinte (e-fis-g-ab). Es folgen noch zwei weitere Präsentationen des Themenbeginns (T. 273 und T. 277), deren erste jetzt die Transpositionsstufe und Harmonisierung aus dem 3. Satz (von F-Dur aus und mit den Sekunden a-h-c-d-dis) aufgreift.

Alle bisher angeführten Befunde sind so offenkundig, dass über die Intentionen des Komponisten eigentlich keine Zweifel aufkommen können. Dies ist bei der Stelle, auf die ich jetzt zu sprechen komme und derentwegen ich im Titel dieses Aufsatzes die Frage »Absicht oder Zufall? " gestellt habe, anders. Es geht um jene schon mehrfach angesprochene Zitatstelle im Finale, deren Terzen - so die allgemeine Auffassung - auf den Anfang der Sinfonie zurückverweisen. Nun kann man sich aber fragen, warum Brahms, wenn er schon auf das Thema des ersten Satzes anspielen wollte, dieses nicht auf der originalen Transpositionsstufe tat. Im Thema wird die Terzenkette von der Quinte aus begonnen ( $h$-g-e-c ...), während das Zitat mit der I. Stufe anfängt (e-c-a-fis ...). Wie Beispiel 4 zeigt, hätte Brahms die originale Transpositionsstufe wählen können (vgl. das System «Konstrukt ), ohne den übrigen Tonsatz der 29. Variation (in der das Zitat ja zum ersten Mal erklingt) verändern zu müssen. Es sei noch einmal daran erinnert, dass das eigentliche Chaconne-Thema e-fis-g-a-ais- $h$... in dieser Variation nicht als reale Stimme vorkommt, sondern nur indirekt aus den Tönen der Terzenkette hervorscheint (vgl. die Notennamen im 2. und 3. System). Auch sonst gibt es im 4. Satz Variationen, in denen das Thema gesucht werden muss, weil es in Diminutionen verborgen ist, in wechselnden Stimmen und Instrumenten erklingt oder im Takt nachhinkend gebracht wird usw. Auch bei meinem sKonstrukt in Beispiel 4, das ja die originale Transpositionsstufe des Hauptthemas im ersten Satz restituiert, wäre die Sekundfolge e-fis-g-a ... mit je einem Schritt pro Takt gegeben, wenngleich immer erst auf dem letzten Achtel (Beispiel 4).

Die von Brahms für das Zitat gewählte Transpositionsstufe der Terzenkette (e-c-afis ...) könnte eventuell mit Blick auf das Hauptthema des ersten Satzes erklärt werden, wo sie vom dritten Ton ab ([h-g]-e-c-a-fis ...) erscheint. Ich möchte hier aber eine andere Erklärung ins Spiel bringen, die bisher noch nicht diskutiert worden ist. Es geht um die Vorbereitung der Reprise im ersten Satz, in deren Verlauf der halb verminderte Vierklang e-c-a-fis eine wichtige Rolle spielt (erstmals T. 240).

Die Vorbereitungspartie zur Reprise umfasst insgesamt 20 Takte. Sie ist als Sequenz angelegt (vgl. Beispiel 5). Eine Variante des Kopfmotivs - Wechselnotenfigur in Achteln aus Takt 9 - wird (bei gelegentlichen Oktavversetzungen) mit einer identisch rhythmisierten Dreiklangsfigur verbunden, die einen voll verminderten Vierklang ergibt: z. B. 


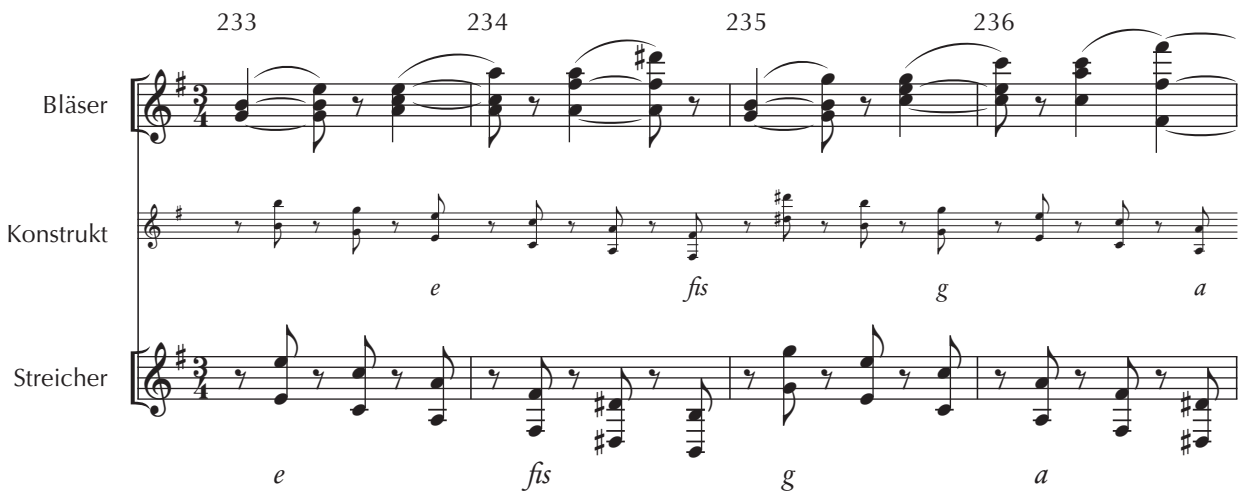

Beispiel 4: Johannes Brahms, 4. Sinfonie e-Moll op. 98, Finale, T. 233-236

Takt 227-230: cis-his-cis-ais/des-b-g-e. Die harmonische Progression, die dem Motivspiel unterlegt ist, verbindet verschiedene Septakkorde miteinander. Das so gewonnene, erst vier-, dann zweitaktige Sequenzglied wird nun in Ganztonschritten aufwärts getrieben. Bezogen auf die Anfangstöne der Motivkette zeigt sich ein Anstieg von des in Takt 227 über es, f, g, a bis zum $h$ in Takt 246. Auf dieser Stufe, die in Form dreier leerer Oktaven erklingt, setzt endlich die Reprise ein, wobei das Thema rhythmisch augmentiert und egalisiert erscheint (Beispiel 5).

Für die Frage nach der Transpositionsstufe des halb verminderten Vierklangs e-c-afis, mit dem Brahms das Terzenzitat im vierten Satz beginnen lässt, ist nun der Umstand überaus wichtig, dass in der Repriseneinleitung des ersten Satzes ab Takt 240 der halbverminderte Terzquartakkord c-e-fis-a (in ebendieser Lage und in subdominantischer Funktion ${ }^{12}$ ) erklingt. Ihm folgt dann in Takt 246 der ganzverminderte Vierklang c-disfis-a, der Teil des Dominantseptnonenakkords über $h$ ist. Eigentlich hätte an der Stelle in Takt 240 der Gis-Akkord (his-dis-fis-gis) zu erscheinen, würde die Sequenz regulär weitergeführt. Das Ausscheren aus dem Sequenzgang wirkt wie ein Signal, mit dem wir auf eine von Brahms möglicherweise intendierte Verknüpfung zwischen erstem und viertem Satz der Sinfonie hingewiesen werden. Diese Bezugnahme - würde sie sich denn bestätigen - ginge viel weiter als das längst bekannte Themenzitat.

Folgende Deutungshypothese möchte ich zur Diskussion stellen: Der Kopfsatz in Sonatensatzform und das als Chaconne/Passacaglia gestaltete Finale ${ }^{13}$ sind nicht nur über ein Themenzitat, sondern auch und vor allem durch zwei parallel geschaltete Sektionen miteinander verbunden (vgl. Bsp. 6). Im ersten Satz handelt es sich um die Takte kurz vor und kurz nach dem Repriseneintritt (T. 240-259), im vierten Satz sind es die Variationen 29 und 30 (T. 233-252). Legt man die Takte, in denen - in beiden Sätzen! - erstmals

12 Der Liegeklang fis-a-c-e ist Subdominante von e-Moll. In Takt 245 tritt dann ganz leise ein dominantisches $H$ in den Bässen hinzu, gefolgt vom Dominantseptnonenakkord von e-Moll. Die Tonika wird danach von den Tönen des Themas repräsentiert ( $h$-g-e), die aber unharmonisiert bleiben, um dann dem Tonika-Gegenklang (C-Dur) in Takt 249 Platz zu machen.

13 Vgl. zur Frage, welche Komposition als Vorlage für Brahms' Finale gedient hat, Petersen 2013. 

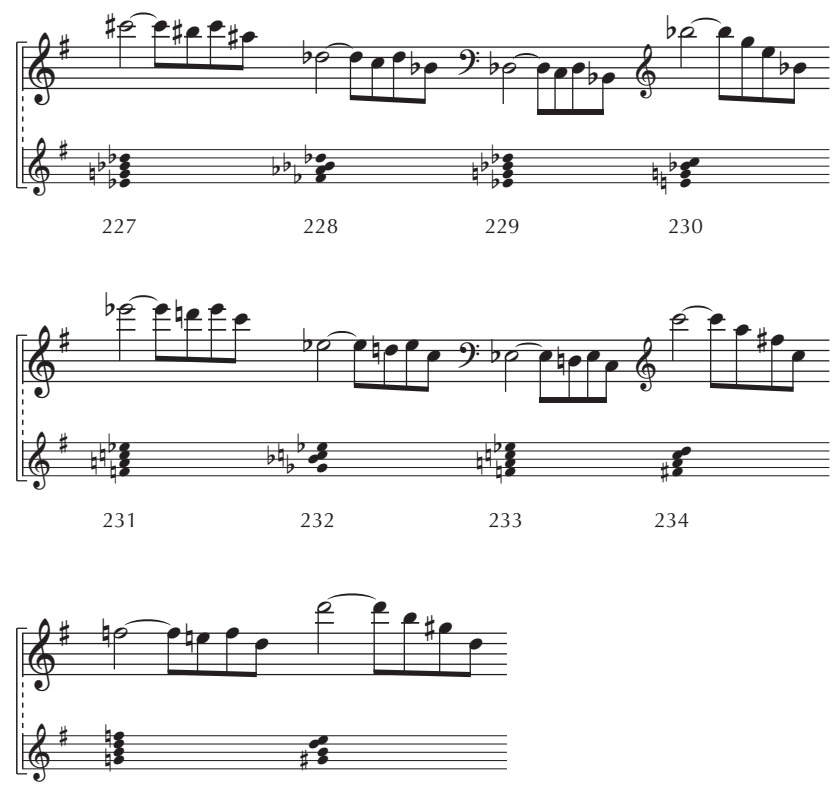

235236

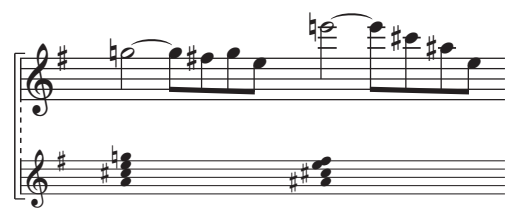

237
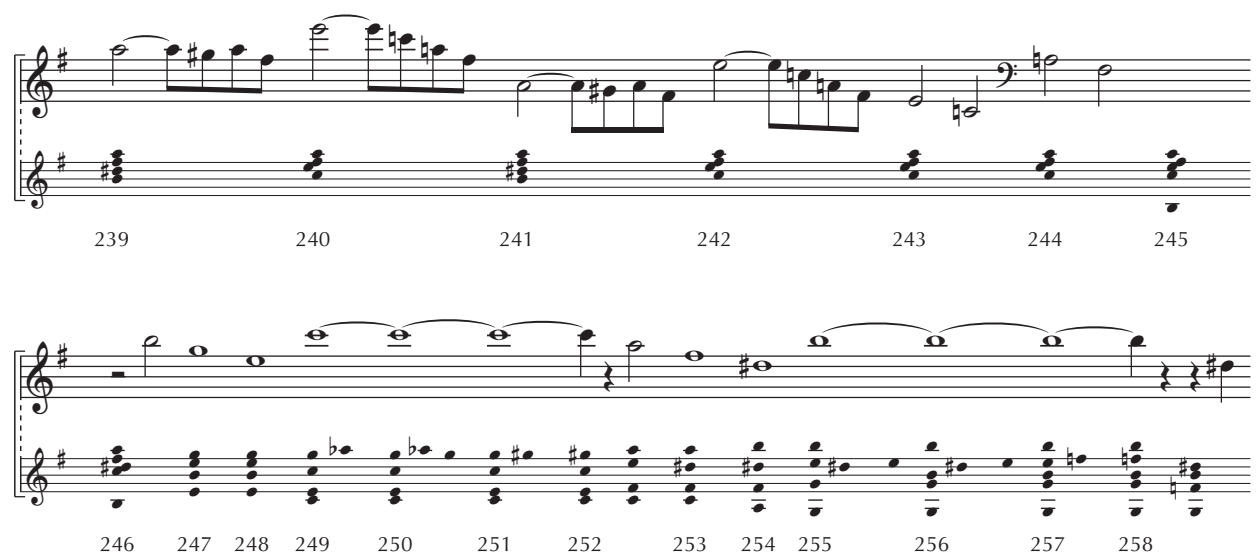

Beispiel 5: Johannes Brahms, 4. Sinfonie e-Moll op. 98, 1. Satz, Einleitung der Reprise (Auszug), T. $227-258$ 
der gebrochene Terzenvierklang e-c-a-fis erscheint, untereinander und verfolgt von da an über die sich anschließenden 20 Takte den Verlauf beider Sätze (T. $240=$ T. 233; $2 / 2=3 / 4$ ), dann ergeben sich signifikante Übereinstimmungen an markanten Punkten (Beispiel 6).

Das Notenbeispiel ist so angelegt, dass in den beiden Akkoladen (oben für den 1., unten für den 4. Satz) die Terzenprogressionen zu erkennen sind. Der übrige Tonsatz, sowie alle sekundären Komponenten wie Lautstärke, Klangfarbe, Artikulation, Phrasierung usw. sind ausgeblendet. Zwischen den Akkoladen stehen die Taktzahlen, anhand derer die Terzengänge auf die vollständige Partitur zu beziehen sind.

Folgende Sachverhalte scheinen mir bemerkenswert, weil sie die oben dargelegte Hypothese zu stützen vermögen:

- Den Takten vor Eintritt der Reprise im ersten Satz, die ganz von dem halb verminderten, gebrochenen Vierklang e-c-a-fis ausgefüllt sind (T. 240-244), entsprechen im Finale Terzenketten mit den Anfangstönen e-c-a-fis ... (T. 233ff.). Die weiterführenden Terzenstufen dis, $h$ und $g$ sind außerdem so platziert, dass sie bei einem virtuellen Zusammenklang der beiden Passagen mit den Terzen des ersten Satzes konsonieren würden.

- Beim Eintritt des (augmentierten) Hauptthemas im ersten Satz wird der Grundton E erst verzögert erreicht. Im Finale ıwarten` nun die Terzenketten der 30. Variation so lange, bis deren Initialton und der Themengrundton zusammen erscheinen (1. Satz: T. 248, 4. Satz: T. 241). Auch hier ist festzuhalten, dass die (flinken) Terzen des Finales zu den (gedehnten) Tönen des Kopfsatzes passten, wollte man sie denn zusammendenken.

- Auch bei der zweiten Teilphrase der Themenmelodie des ersten Satzes (T. 252), die mit den Tönen a-fis-dis- $h$ beginnt, swarten die Terzen im Finale wiederum bis zum dis des Hauptthemas, das dann sozusagen Stichwortgeber für das dis der verlängerten Terzenkette dis-h-g-e-c in der 30. Variation ist (4. Satz: T. 247-252). Selbst hier würden sich die Terzenfolgen beider Sektionen im virtuellen Zusammenklang svertragen`.

Es ist oft bedauert worden, dass Brahms seine Skizzen vernichtet hat, weil er Einblicke in seine Komponierwerkstatt ausschließen wollte. Versuche, trotzdem hinter die Geheimnisse seines Schaffens zu kommen, sind deshalb leicht dem Verdacht ausgesetzt, spekulativ zu sein. Dem können Analytiker und Interpreten nur entgegenwirken, indem sie ihre Deutungsvorschläge sorgfältig begründen. Wenn diese auch kaum einmal den Status eines wissenschaftlichen Beweises erlangen mögen, so werden sie doch mehr oder weniger plausibel sein. Im hier diskutierten Fall scheinen mir die Befunde zu den diatonischen Sekunden-, Terzen- und Quartenzirkeln in Brahms' 4. Sinfonie Evidenz zu besitzen. Die weiterführende und sicherlich ıgewagte` Annahme, dass der Komponist bei der Arbeit am Finale sich den bereits vorliegenden ersten Satz herbeigeholt habe, um 

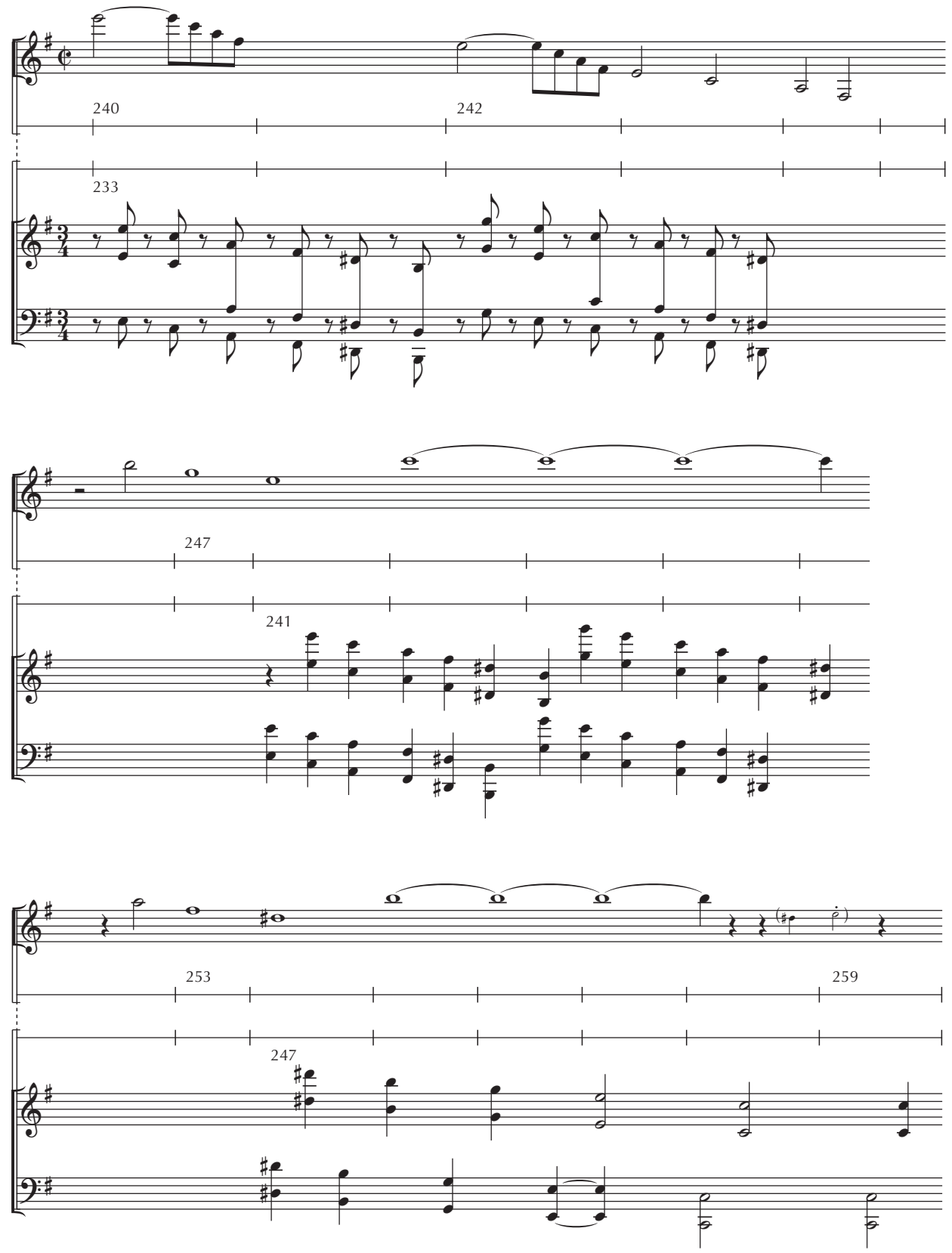

Beispiel 6: Johannes Brahms, 4. Sinfonie e-Moll op. 98, Terzenstruktur des Hauptthemas im Umkreis der Reprise im 1. Satz (T. 240-259) und freie Wiederholung der Sektion im 4. Satz (T. 233-252) 
die letzten beiden Variationen ${ }^{14}$ Takt für Takt mit der Reprisen-Sektion des Kopfsatzes abgleichen zu können, wird womöglich auf Skepsis stoßen. Auf die seltsamen Parallelen hingewiesen und damit der Auffassung, im Finale würde der `Anfang` der Sinfonie ızitiertı, widersprochen zu haben, war mir aber ein Anliegen. Nicht auf den Sinfoniebeginn, sondern auf die Reprise des ersten Satzes deutet Brahms im Finale zurück.

\section{Literatur}

Finscher, Ludwig (1990), „Arnold Schönbergs Brahms-Vortrag", in: Neue Musik und Tradition. Festschrift Rudolf Stephan zum 65. Geburtstag, hg. von J. Kuckertz u.a., Laaber: Laaber, 485-500.

Heuß, Alfred (1907-08), »Eine Händel-Beethoven-Brahms-Parallele», in: Die Musik 7, 147-151.

Horton, John (1968), Brahms Orchestral Music, London: BBC.

Jacobson, Bernard (1977), The Music of Johannes Brahms, London: Tantivy Press.

Kalbeck, Max (1904-1914), Johannes Brahms, 4 Bde., Wien und Leipzig: Dt. BrahmsGesellschaft.

Knapp, Raymond (1997), Brahms and the Challenge of the Symphony, New York: Pendragon.

Kross, Siegfried (1997), Johannes Brahms. Versuch einer kritischen Dokumentar-Biographie, 2 Bde., Bonn: Bouvier.

Mäckelmann, Michael (1991), Johannes Brahms. IV. Symphonie E-Moll Op. 98 (= Meisterwerke der Musik 56), München: Fink.

Petersen, Peter (2013), „Das Variationen-Finale aus Brahms' e-Moll-Sinfonie und die c-Moll-Chaconne von Beethoven (WoO 80)«, AfMw 70, 105-118.

Riemann, Hugo, (1897), »Johannes Brahms. IV. Symphonie (E-moll) op. 98«, in: Johannes Brahms. Symphonien und andere Orchesterwerke (= Meisterführer Nr. 3), erläutert von I. Knorr, H. Riemann, J. Sittard nebst einer Einleitung: Johannes Brahms' Leben und Schaffen, von A. Morin, Berlin: Schlesinger, 77-93.

Schmidt, Christian Martin (1971), Verfahren der motivisch-thematischen Vermittlung in der Musik von Johannes Brahms, dargestellt an der Klarinettensonate f-moll, op. 120,1, München: Katzbichler.

Vetter, Walter (1913-14), „Der erste Satz von Brahms' E-Moll-Symphonie. Ein Beitrag zur Erkenntnis moderner Symphonik«, in: Die Musik 13, 3-15, 83-92 und 133-145.

Walker, Alan (1962), A Study in Musical Analysis, London: Barrie and Rockliff.

14 Die 30. und letzte Variation ist um vier Takte verlängert, hat also statt der bis dahin geltenden acht jetzt zwölf Takte. 